\title{
Recognition memory deficits in mild cognitive impairment
}

\author{
Salvador Algarabel ${ }^{1}$, Manuel Fuentes ${ }^{1}$, Joaquín Escudero², \\ Alfonso Pitarque ${ }^{1}$, Vicente Peset ${ }^{2}$, José-Francisco Mazón ${ }^{2}$, \\ and Juan-Carlos Meléndez ${ }^{1}$ \\ ${ }^{1}$ University of Valencia, Valencia, Spain \\ ${ }^{2}$ Neurology Service, General Hospital, Valencia, Spain
}

\begin{abstract}
There is no agreement on the pattern of recognition memory deficits characteristic of patients diagnosed with mild cognitive impairment (MCI). Whereas lower performance in recollection is the hallmark of MCI, there is a strong controversy about possible deficits in familiarity estimates when using recognition memory tasks. The aim of this research is to shed light on the pattern of responding in recollection and familiarity in MCI. Five groups of participants were tested. The main participant samples were those formed by two MCI groups differing in age and an Alzheimer's disease group (AD), which were compared with two control groups. Whereas one of the control groups served to assess the performance of the MCI and $\mathrm{AD}$ people, the other one, composed of young healthy participants, served the purpose of evaluating the adequacy of the experimental tasks used in the evaluation of the different components of recognition memory. We used an associative recognition task as a direct index of recollection and a choice task on a pair of stimuli, one of which was perceptually similar to those studied in the associative recognition phase, as an index of familiarity. Our results indicate that recollection decreases with age and neurological status, and familiarity remains stable in the elderly control sample but it is deficient in MCI. This research shows that a unique encoding situation generated deficits in recollective and familiarity mechanisms in mild cognitive impaired individuals, providing evidence for the existence of deficits in both retrieval processes in recognition memory in a MCI stage.
\end{abstract}

Keywords: Mild cognitive impairment; Alzheimer's disease; Recollection; Familiarity; Recognition memory.

This research was supported by Grant PSI2010-17425 from the 'Ministerio de Ciencia e Innovación (Spanish Ministry of Science and Innovation)' and FEDER funds from the European Community.

Address correspondence to: Salvador Algarabel, Facultad de Psicologia, Universidad de Valencia, Blasco Ibanyez, 21, E46010 Valencia, Spain. E-mail: salvador.algarabel@uv.es 
Mild cognitive impairment (MCI) is an interim state that frequently anticipates dementia. The first symptoms begin when relatives of the patient notice episodic memory lapses that while not interfering with daily life become significant. Current research has focused on studying these memory deficits with the aim of anticipating as accurately as possible who will develop dementia. Although these memory deficits can be estimated with laboratory tests such as free or cued recall, recognition paradigms are simpler to use and enable the estimation of two independent episodic memory components simultaneously: recollection and familiarity, which may be of importance for characterizing these memory deficits.

Recollection underlies the retrieval of memory facts in which we can give contextual details of their original encounter. Familiarity, on the other hand, is a more automatic process that gives rise to the feeling of 'déjà vu' devoid of any associated details that would allow us to place it in the past. Whereas recollection diminishes severely with age and dementia, many researchers defend the view that familiarity remains stable (e.g., Bastin \& Van der Linden, 2003; Fleischman, Wilson, Gabrieli, Bienias, \& Bennett, 2004; McCabe, Roediger III, McDaniel, \& Balota, 2009; but see Prull, Dawes, Martin III, Rossenberg, \& Light, 2006). The presence of deficits in familiarity is controversial in people diagnosed with MCI. Previous studies that assessed familiarity in MCI patients produced inconclusive results. Initially, Westerberg, Paller, Holdstock, Mayes, and Reber (2006) concluded that familiarity was intact in MCI (see also Anderson et al., 2008; Hudon, Belleville, \& Gauthier, 2009; Serra et al., 2010), although subsequent reports (Algarabel et al., 2009; Ally, McKeever, Waring, \& Budson, 2009; Wolk, Signoff, \& DeKosky, 2008) did contradict Westerberg's initial negative findings. These contradictory results may be due largely to methodological problems associated with the use of experimental paradigms that are based on premises not fully accepted (see e.g., Wixted, Mickes, \& Squire, 2010). More specifically, Westerberg et al. (2006) estimated familiarity from a fouralternative choice task in which one of the stimuli was the studied one and the lures were slight variations of it. Their assumption was that in the task familiarity had to be the process used in responding given that by recollection you could not obviously discriminate the correct from the incorrect stimuli given their similarity. This is an arguable assumption (see e.g., Khoe et al., 2000) but an additional significant methodological point is that the null difference between MCI and control groups was based on very small samples (8 individuals in the each group), which obviously lack statistical power.

Our basic purpose in this paper is to explore recollection and familiarity processes simultaneously in MCI participants, avoiding the methodological problems that have troubled previous research reports. This will be accomplished with a single study situation, to keep encoding conditions constants, to be followed by a recollection and a familiarity test. The study phase involved 
studying a series of word pairs that had to be associated, simultaneously creating a perceptual fluency feeling. In the recollection test (associative recognition), participants had to distinguish unchanged, from rearranged word pairs. To be successful in this test, people have to recover the original studied association and this is why it is considered an excellent paradigm for estimating recollection (e.g., Wixted et al., 2010). The familiarity test was a two-forced choice test in which none of the words have been studied but one of them elicited the same perceptual fluency generated in the study phase. If participants are led to believe, and this fact becomes completely essential in the design, that one of the words has been studied, the word eliciting familiarity will be chosen as the 'studied' word, and can be taken as an index of the degree of familiarity induced in the study phase (see Algarabel, Pitarque, Tomás, \& Mazón, 2010; Parkin et al., 2001). We have used this estimation of the familiarity processes before (Algarabel et al., 2009, 2010) very successfully because it does not require making any strong assumption about the nature of familiarity (see Wixted et al., 2010) as other procedures do.

Regarding our predictions, we expected to observe a clear deficit in recollection in patients with $\mathrm{AD}$ or those with MCI given that this is the episodic mechanism constantly shown to be deficient in dementia. Nevertheless, the most interesting predictions concern performance in the familiarity test. As indicated previously, this familiarity test was a two-alternative choice test in which the subjects were led to believe that one of the words had already been studied. If a deficit in familiarity is to be found, those participants with MCI and AD should show lower preference for perceptually related new words in the choice test, than the controls. Finally, if choice of truly studied words draws also more heavily on familiarity than in recollection, we expected a similar response pattern in these trials to those based on pairs of completely new words in the healthy groups but not to the 'clinical' ones. With this design, we try to establish more firmly the pattern of episodic memory deficits associated with aging, and particularly with the development of dementia.

\section{METHODS}

\section{Participants}

The experiment was made up of five groups; 12 young healthy volunteers, 21 healthy elderly adults, 20 multiple-domain younger amnestic patients, 20 multiple-domain older amnestic patients and $8 \mathrm{AD}$ diagnosed patients. The younger MCI group was matched to the older adult healthy controls, whereas the older MCI group was matched to the AD group (see Table 1 for statistical contrast of the demographics variables) in education (the older adults), age and gender, although most participants in the AD 


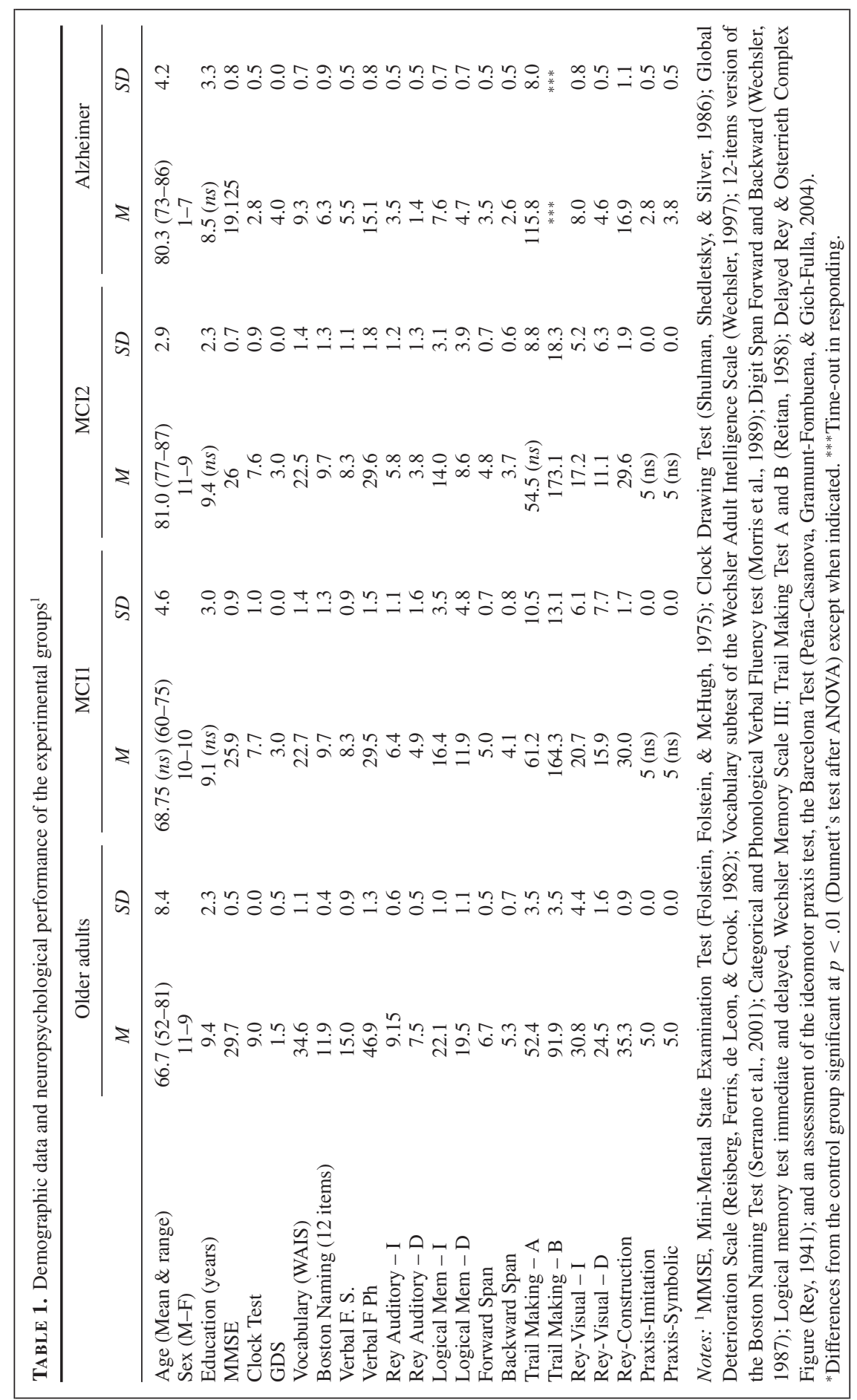


group were women. The healthy elderly were cognitively normal volunteers, most of whom were partners of patients who had been referred to the Neurology Unit of the General Hospital of Valencia (Spain) for evaluation. Diagnosis was the end-result of an extended evaluation, including medical history, physical, neurological, psychiatric, and neuropsychological assessment (see Table 1 for some detailed data). Initially, none of the MCI patients received pharmacological treatment. AD patients were diagnosed according to NINCDS-ADRDA criteria and criteria established by the Spanish Neurological Society (McKhann et al., 1984). All AD patients received acetylcholinesterase inhibitors. Exclusion criteria for patients were: significant asymptomatic neurovascular alteration disease confirmed by brain MRI, a positive Hachinski ischemic scale value of 4 or higher, a history of previous symptomatic stroke, any medical condition significantly affecting the brain, serious psychiatric symptoms or a history of drug abuse. Patients included in the MCI sample met the diagnostic criteria specified by Petersen (2004) as multiple domain amnestic type. Cut-off scores for abnormality in each neuropsychological test were $1.5 \mathrm{SD}$ s below the mean score for the corresponding age and education group. All participants gave written informed consent for the study, which was approved by the institutional review boards of the Valencia General Hospital and the University of Valencia, Spain.

\section{Materials and Design}

The stimuli were 520 Spanish words drawn from a frequency dictionary (Alameda \& Cuetos, 1995) of between three and nine letters in length. As indicated in the introduction, perceptual fluency was created by selecting words with specific letter combinations. For this purpose, half of the words had the letter 'a' but not 'o' (list A), and the other half had the opposite letter composition (list $\mathrm{O}$ ). Both lists were equated in length and mean frequency per two million (frequency $=94.68$, length $=5.50$ letters, and 93.73 and 5.49 for list A and O, respectively). The experiment consisted of ten studytest lists in which word pairs were presented for study and left either intact or were rearranged for testing. Therefore, each participant was exposed to 10 study-test cycles in the associative recognition phase, to be followed by a final choice test. All pairs (both members of each) were extracted from list $\mathrm{A}$ or from list $\mathrm{O}$ in a counterbalanced way across subjects. Both types of stimuli were never mixed at study for any individual participant. In each block, 10 word pairs were studied but only 6 were tested, leaving the remaining words for the final choice test, except the first and the last one in the list that served as fillers and were never tested. Each recognition test consisted of presenting 3 intact and 3 rearranged pairs for which every participant had to press the keys corresponding to the letters 'd' (rearranged pair) and 'k' (identical pair) on the computer keyboard. Once the associative recognition test was finished, a 60 two-word choice test was given, in which participants had 
to select the word previously studied. Half of these pairs (30) paired a studied word with a new word from the same type of list (either list A or list O). The other half (30) presented pairs of completely new words that were never presented, but in which one of them was extracted from the same type of list to which the participant was exposed when studying, and the other word from the alternative list. That is, if participants had studied stimuli from list A, the 'new' words being chosen paired one word from list A (although the specific word was not studied) with one from list $\mathrm{O}$ which was also never studied. The experiment was run individually with words presented side by side for $3500 \mathrm{~ms}$ in the center of a laptop screen controlled by e-prime software (Schneider, Eschman, \& Zuccolotto, 2002). Participants had to indicate 'studied' or 'new' in the choice test, pressing the keys corresponding to the letters ' $\mathrm{d}$ ' and ' $\mathrm{k}$ ', respectively. Once the experiment was completed, participants were asked about the techniques they had used to memorize the words in the study task with the aim of identifying participants who had noticed anything related to the letter composition of the words studied in the experiment.

\section{Statistical Analyses}

Demographic data and neuropsychological test performance were analyzed by means of a single factor (group) between subject ANOVA followed by Dunnet's tests when appropriate, in which all groups were tested against the older adults. The assumption about the homogeneity of variances in the analysis of variance was tested calculating Levene's test. The associative recognition performance was analyzed by means of an ANOVA with groups as an independent variable on hits minus false alarms, which is a non-parametric discrimination index of recognition performance, followed by an independent analysis of hits and false alarms. Hits are defined as the proportion of times in which a pair of stimuli were presented intact and recognized as such. False alarms, on the other hand, are the proportion of times in which rearranged pairs of stimuli are recognized (wrongly) as originally presented together at study. More concretely, and as an example, if the pair 'dog-chair' were presented at study and at test a participant answered 'yes', then this is a hit. However, if 'dog-pair' was never jointly presented, and at test, a participant said 'yes' ('it was'), then this is a false alarm. Choice trials were analyzed by an ANOVA with groups as an independent variable followed by a Tukey's test. For specific comparisons between means, independent sample $t$ tests were used.

\section{RESULTS}

The neuropsychological data show a pattern of deficits coherent with the nature of the different groups and in accordance with those of other published reports. In particular, the MMSE, the GDS, and the Clock Drawing 
Test showed significant differences between patients and the healthy adult controls, whereas the older MCI and the Alzheimer's group showed the worst performance. The MCI and $\mathrm{AD}$ groups showed gradual and significant deficits in memory (working, verbal, and visual memory), attention, language and visuo-spatial and visuo-constructive abilities. Only those with AD were deficient in the Praxis tests. Complete detailed information about the patients' neuropsychological performance can be found in Table 1.

None of the participants noticed anything special about the letter composition of the presented words. The proportion of 'intact pairs' (associative recognition) or 'old' words (choice) is summarized in Table 2.

To analyze associative recollection, a between subjects ANOVA was performed on hits minus false alarms. The ANOVA revealed a significant effect of Groups, $F(4,75)=44,671$, MSE $=0.018, p<.001$. Given that Levene's test of homogeneity of variances was significant, $p=.024$, we calculated Dunnett's T3 post-hoc test. The test indicated that the young group had better associative recognition memory than the rest, followed by the older adults control group $(p<.01)$, and finally the patient groups which did not differ among them. That is, the $\mathrm{MCI}$ and the $\mathrm{AD}$ groups performed at floor level. These significant effects were entirely due to the differences in false alarms, $F(4,75)=8.51$, MSE $=0.059, p<.001$, and not hits, $F(4,75)=1.687, \mathrm{MSE}=0.048, p=.162$. In this last case, Levene's test was also significant $(p=.018)$.

TABLE 2. Mean proportions and standard deviations $(S D)$ for the different tasks and conditions of the experiment as a function of group and $\operatorname{task}^{1}$

\begin{tabular}{|c|c|c|c|c|c|c|c|c|c|c|}
\hline \multirow[b]{3}{*}{ Group } & \multicolumn{6}{|c|}{ Associative recognition } & \multicolumn{4}{|c|}{ Choice } \\
\hline & \multicolumn{2}{|c|}{ Hits } & \multicolumn{2}{|c|}{ False alarms } & \multicolumn{2}{|c|}{$\begin{array}{l}\text { Hits-false } \\
\text { alarms }\end{array}$} & \multicolumn{2}{|c|}{ Studied } & \multicolumn{2}{|c|}{ New } \\
\hline & $M$ & $S D$ & $M$ & $S D$ & $M$ & $S D$ & $M$ & $S D$ & $M$ & $S D$ \\
\hline Younger adults ${ }^{(\mathrm{a})}$ & 0.79 & 0.14 & $0.16^{\text {bcde }}$ & 0.11 & $0.62^{\text {bcde }}$ & 0.21 & $0.87^{\text {cde }}$ & 0.10 & 0.63 & 0.09 \\
\hline Older adults $^{(\mathrm{b})}$ & 0.77 & 0.19 & $0.57^{\mathrm{a}}$ & 0.22 & $0.20^{\text {acde }}$ & 0.12 & $0.80^{\mathrm{de}}$ & 0.12 & 0.64 & 0.14 \\
\hline $\mathrm{MCI}^{(\mathrm{c})}$ & 0.70 & 0.21 & $0.62^{\mathrm{a}}$ & 0.27 & $0.08^{\mathrm{ab}}$ & 0.12 & $0.73^{\text {ade }}$ & 0.12 & 0.56 & 0.13 \\
\hline $\mathrm{MCI} 2^{(\mathrm{d})}$ & 0.64 & 0.26 & $0.62^{\mathrm{a}}$ & 0.27 & $0.02^{\mathrm{ab}}$ & 0.11 & $0.64^{\mathrm{abc}}$ & 0.10 & 0.56 & 0.12 \\
\hline $\mathrm{AD}^{(\mathrm{e})}$ & 0.60 & 0.26 & $0.56^{\mathrm{a}}$ & 0.28 & $0.04^{\mathrm{ab}}$ & 0.10 & $0.55^{\mathrm{abc}}$ & 0.08 & 0.51 & 0.13 \\
\hline \multicolumn{11}{|c|}{$\begin{array}{l}\text { Notes: }{ }^{1} \text { In this table, a hit is a response given to studied pair of words left intact at test. A false } \\
\text { alarm is a response of 'intact' when at test the word pair appeared rearranged. In the choice test, } \\
\text { 'studied' indicates the proportion of trials in which participants identified the originally presented } \\
\text { word, whereas 'new' is the proportion of times in which the word perceptually related to the study list } \\
\text { but never presented, was chosen as 'studied'. The letter code is introduced to indicate the significant } \\
\text { contrasts (at least } p<.05 \text { ) between each group and the rest. }\end{array}$} \\
\hline
\end{tabular}


The ANOVA on choice for the studied words revealed a significant group effect, $F(4,75)=16.91$, MSE $=0.011, p<.001$, as expected. Follow-up post-hoc tests (Tukey's test) revealed that there was a progressive decline in remembering between the performance levels of the two control groups on the one hand and the MCI and AD samples. Finally, the ANOVA on choice for new words in which one of them belonged to the study letter set revealed a main effect of group, $F(4,75)=2.52$, MSE $=0.039, p=.048$. Given the considerable expectations we had in the performance of the two control groups (no difference in familiarity), on the one hand, and the two MCI groups (similar deficit in familiarity) we decided to contrast the possible existence of a familiarity deficit with a more powerful test. With this purpose we added up the two controls on the one hand and the two MCI groups, on the other. This test of a single control against a single MCI group (one tailed) revealed a significant difference between both, $t(70)=2.68, S E M=0.029$, $p<.01$.

\section{DISCUSSION}

The statistical analyses revealed that the young adults outperformed the older adults who, in turn, did better than the two MCI and the AD samples in the associative recognition test. This is the classic decrease in episodic memory function found with age and dementia. Associative recognition is a similar test to other explicit tests, such as free recall, in showing differences in retrieval capabilities. The choice test on new items revealed no differences in familiarity between the two control groups, as expected if the test is a correct indicator of familiarity, and a further confirmation of inferior performance in the patient groups, particularly the MCI patients, irrespective of age. It is thus important to realize that the fact that perceptual familiarity remains weak is a testimony to its subconscious nature. If the participants had noticed the letter repetition pattern, they would have used it systematically, and selection of the 'correct' word would have increased considerably. Finally, the choice test on studied items was not sensitive to differences in explicit retrieval mechanisms with age, and shows no difference between the two healthy groups. In this respect, choice on the studied and new items gave similar results. It is safe to assume that the choice test is the equivalent to a classic yes-no recognition test in which response is the result of a mixture of recollection and familiarity with heavy emphasis on familiarity. Given that there are no deficits in familiarity between the two healthy adult groups, the older participants were able to compensate their deficits in recollection with the help of familiarity. The present experiment lends strong support to the associative deficit hypothesis (Naveh-Benjamin, 2000; Old \& Naveh-Benjamin, 2008), which states that it is the capability to link independent pieces of information that becomes deficient with age. Although in this experiment test time and format were not 
equated, group differences showed up in the associative recognition and not in the choice test. If we take into account the fact that the associative test is immediate and the choice test is delayed, the result is an even stronger endorsement of the hypothesis.

The present research further supports the presence of a familiarity deficit in cases of mild cognitive impairment. Originally, Westerberg et al. (2006) claimed that familiarity was preserved in MCI patients. They based their conclusion on a comparison between MCI and control patients in choice tests of very similar stimuli in which they found no differences between the two groups. This conclusion, as well as others obtained using the remember-know procedure (Hudon et al., 2009; Serra et al., 2010), was based on the assumption that this dependent variable was more susceptible to the influence of familiarity than others (but see Migo, Montaldi, Norman, Quamme, \& Mayes, 2009). As in a previous paper (Algarabel et al., 2009) we do not find the assumption to be well founded. Other researchers (Wolk et al., 2008), as well as ourselves (Algarabel et al., 2009), have also provided evidence that there is a deficit in familiarity in these patients who, in many cases, will develop Alzheimer's disease. In this paper, we have also obtained deficits in familiarity and recollection keeping the encoding conditions constant. Finally, one important observation is that the cause of the deficits found in the patient populations is more due to an increase in false alarms than to a decrease in hits, or due to an inverse effect on both indices. Paradoxically, this observation seems to be in direct conflict with the associative deficit hypothesis put forward by Naveh-Benjamin (2000) according to which we should expect more decreases in hits than increases in false alarms with age. Most researchers attribute the increase in false alarms with age to an over-reliance on familiarity. However, in this paper using a purely associative recognition task, the observation of a great increase in false 'recollection' suggests that the possible cause of the effect is not over-reliance on familiarity but an increase in false recollection with age. A recent paper by McCabe et al. (2009) supports this conclusion. These authors, reviewing the literature generated by the remember-know paradigm, observed that older adults had more than double the level of 'remember' false alarms than younger adults and at the same time the effect was very small in 'knowing' false alarms. Moreover, they found an association between false remembering and frontal lobe functioning tests and between 'remember' hits and temporal lobe functioning tests, leading one to believe that instead of an over-reliance on familiarity, these results can be better explained assuming that old adults make misattributions or false recollections with items not studied before.

In conclusion, this paper contributes to extend the knowledge about the memory deficits characterizing mild cognitive impairment individuals. These episodic memory deficits (see Dubois et al., 2007, 2010) constitute, at the moment, the main predictors of conversion to AD. However, within these 
memory deficits retrieval deficiencies as measured by free or delayed cued recall have been the exclusive consideration up to this point. Only recently the exploration of possible deficits in more automatic forms of memory have been explored. This paper documents these deficits in those people diagnosed with mild cognitive impairment. We have also documented the sensitivity of the associative recognition task, long time used in experimental settings, to detect episodic memory deficits, not only produced in dementia states, but with the advancement of age. Further research needs to be carried out exploring the possibility that the deficits in perceptual familiarity extends to other forms of familiarity, particularly familiarity based in semantic content. It is well known that MCI and AD patients have also deficits in semantic memory. The research on deficits in familiarity based on semantic content would help to complete the characterization of these types of dementia.

\footnotetext{
Original manuscript received 11 April 2011 Revised manuscript accepted 20 October 2011 First published online 17 January 2012
}

\section{REFERENCES}

Alameda, J. R., \& Cuetos, F. (1995). Diccionario de frecuencias de las unidades lingüísticas del castellano [Frequency dictionary of the linguistic units of Spanish]. Universidad de Oviedo, Departamento de Psicología, Oviedo.

Algarabel, S., Escudero, J., Mazón, J. F., Pitarque, A., Fuentes, M., Peset, V., et al. (2009). Familiarity-based recognition in the young, healthy elderly, mild cognitive impaired and alzheimer's patients. Neuropsychologia, 47, 2056-2064.

Algarabel, S., Pitarque, A., Tomás, J., \& Mazón, J. F. (2010). Explorations of familiarity produced by words with specific combinations of letters. European Journal of Cognitive Psychology, 22, 265.

Ally, B. A., McKeever, J. D., Waring, J. D., \& Budson, A. E. (2009). Preserved frontal memorial processing for pictures in patients with mild cognitive impairment. Neuropsychologia, 47, 2044-2055.

Anderson, N. D., Ebert, P. L., Jennings, J. M., Grady, C. L., Cabeza, R., \& Graham, S. J. (2008). Recollection- and familiarity-based memory in healthy aging and amnestic mild cognitive impairment. Neuropsychology, 22, 177-187.

Bastin, C., \& Van der Linden, M. (2003). The contribution of recollection and familiarity to recognition memory: A study of the effects of test format and aging. Neuropsychology, 17, 14-24.

Dubois, B., Feldman, H. H., Jacova, C., DeKosky, S. T., Barberger-Gateau, P., Cummings, J., et al. (2007). Research criteria for the diagnosis of Alzheimer's disease: Revising the NINCDS-ADRDA criteria. The Lancet Neurology, 6, 734-746.

Dubois, B., Feldman, H. H., Jacova, C., Cummings, J. L., DeKosky, S. T., Barberger-Gateau, P., et al. (2010). Revising the definition of Alzheimer's disease: A new lexicon. The Lancet Neurology, 9, 1118-1127. 
Fleischman, D. A., Wilson, R. S., Gabrieli, J. D. E., Bienias, J. L., \& Bennett, D. A. (2004). A longitudinal study of implicit and explicit memory in old persons. Psychology and Aging, 19, 617-625.

Folstein, M., Folstein, S., \& McHugh, P. (1975). 'Mini-Mental State': A practical method for grading the cognitive state of patients for the clinician. Journal of Psychiatric Research, 12, 189-198.

Hudon, C., Belleville, S., \& Gauthier, S. (2009). The assessment of recognition memory using the Remember/Know procedure in amnestic mild cognitive impairment and probable Alzheimer's disease. Brain and Cognition, 70, 171-179.

Khoe, W., Krolla, N. E. A., Yonelinas, A. P., Dobbins, I. G., \& Knight, R. T. (2000). The contribution of recollection and familiarity to yes \pm no and forced-choice recognition tests in healthy subjects and amnesics. Neuropsychologia, 38, 1333-1341

McCabe, D. P., Roediger III, H. L., McDaniel, M. A., \& Balota, D. A. (2009). Aging reduces veridical remembering but increases false remembering: Neuropsychological test correlates of remember-know judgments. Neuropsychologia, 47, 2164-2173.

McKhann, G., Drachman, D., Folstein, M., Katzman, R., Price, D., \& Stadlan, E. M. (1984). Clinical diagnosis of Alzheimer's disease: Report of the NINCDS-ADRDA Work Group under the auspices of Department of Health and Human Services Task Force on Alzheimer's Disease. Neurology, 34, 939-944.

Migo, E., Montaldi, D., Norman, K. A., Quamme, J., \& Mayes, A. (2009). The contribution of familiarity to recognition memory is a function of test format when using similar foils. The Quarterly Journal of Experimental Psychology, 62, 1198.

Morris, J. C., Heyman, A., Mohs, R. C., Hughes, J. P., van Belle, G., Fillenbaum, G., et al. (1989). The consortium to establish a registry for Alzheimer's disease (CERAD), I: Clinical and neurological assessment for Alzheimer's disease. Neurology, 39, 1159-1165.

Naveh-Benjamin, M. (2000). Adult age differences in memory performance. Journal of Experimental Psychology: Learning, Memory and Cognition, 26, 1170.

Old, S. R., \& Naveh-Benjamin, M. (2008). Differential effects of age on item and associative measures of memory: A meta-analysis. Psychology and Aging, 23, 104-118.

Parkin, A. J., Ward, J., Squires, E. J., Furbear, H., Clark, A., \& Townshend, J. (2001). Data-driven recognition memory: A new technique and some data on age differences. Psychonomic Bulletin \& Review, 8, 812-819.

Peña-Casanova, J., Gramunt-Fombuena, N., \& Gich-Fulla, J. (2004). Tests neuropsicológicos. Fundamentos para una neuropsicología clínica basada en evidencias [Neuropsychological tests. Foundations for a clinical neuropsychology based on evidence]. Barcelona, Spain: Masson.

Prull, M. W., Dawes, L. L. C., Martin III, A. M., Rossenberg, H. F., \& Light, L. L. (2006). Recollection and familiarity in recognition memory: Adult age differences and neuropsychological test correlates. Psychology and Aging, 21, 107-118.

Reisberg, B., Ferris, S. H., de Leon, M. J., \& Crook, T. (1982). The Global Deterioration Scale for assessment of primary degenerative dementia. American Journal of Psychiatry, 139, 1136-1139.

Reitan, R. M. (1958). Validity of the trail making test as an indicator of organic brain damage. Perceptual and Motor Skills, 8, 271-276.

Rey, A. (1941). L'examen psychologique dans les cas d'encephalopathie traumatique [Psychological examination of cases of traumatic encephalopathy]. Archives de Psychologie, 28, 286-340.

Schneider, W., Eschman, A., \& Zuccolotto, A. (2002). E-Prime reference guide. Pittsburgh, PA: Psychology Software Tools. 
Serra, L., Bozzali, M., Cercignani, M., Perri, R., Fadda, L., Caltagirone, C., et al. (2010). Recollection and familiarity in amnesic mild cognitive impairment. Neuropsychology, 24, 316-326.

Shulman, K. I., Shedletsky, R., \& Silver, I. (1986). The challenge of time: Clock drawing and cognitive function in the elderly. International Journal of Geriatric Psychiatry, 1,135-140.

Wechsler, D. (1987). The Wechsler Memory Scale - Revised Manual. San Antonio, TX: The Psychological Corporation.

Wechsler, D. (1997). The Wechsler Adult Scale-III Manual. San Antonio, TX: The Psychological Corporation.

Westerberg, C. E., Paller, K. E., Holdstock, J. S., Mayes, A. R., \& Reber, P. J. (2006). When memory does not fail: Familiarity-based recognition in mild cognitive impairment and Alzheimer's disease. Neuropsychology, 20, 193-205.

Wixted, J. T., Mickes, L., \& Squire, L. R. (2010). Measuring recollection and familiarity in the medial temporal lobe. Hippocampus, 20, 1195-1205.

Wolk, D. A., Signoff, E. D., \& DeKosky, S. T. (2008). Recollection and familiarity in amnestic mild cognitive impairment: A global decline in recognition memory. Neuropsychologia, 46, 1965-1978. 